\title{
Threshold dynamics of an SEIR epidemic model with a nonlinear incidence rate and a discontinuous treatment function
}

\section{Da-peng Gao ${ }^{1,2} \cdot$ Nan-jing Huang ${ }^{2}$}

Received: 15 May 2018 / Accepted: 26 October 2019 / Published online: 5 December 2019

(c) The Royal Academy of Sciences, Madrid 2019

\begin{abstract}
We consider a susceptible-exposed-infected-removed (SEIR) epidemic model with a nonlinear incidence rate and a discontinuous treatment function. By applying the LaSalle-type invariance principle for differential inclusions and the Lyapunov theory for discontinuous differential equations, we show that the threshold dynamics are completely described by the basic reproduction number $R_{0}$, under some mild assumptions on the incidence rates and the treatment functions. The disease-free equilibrium state is globally asymptotically stable when $R_{0} \leq 1$ and there is endemic equilibrium state which is globally asymptotically stable when $R_{0}>1$. Furthermore, we prove that the disease described by the SEIR epidemic model will die out in finite time. Finally, four illustrative numerical examples are given to support our theoretical results.
\end{abstract}

Keywords Epidemic model · Latent period - Discontinuous treatment · Nonlinear incidence rate $\cdot$ Basic reproduction number . Asymptotic stability

Mathematics Subject Classification 92B05 $\cdot 34 \mathrm{~A} 36 \cdot 34 \mathrm{D} 23$

This work was supported by the National Natural Science Foundation of China (11371015, 11471230, 11671282), the Natural Science Foundation of Sichuan Provincial Education Department (Grant No. 18ZB0581), the Meritocracy Research Funds of China West Normal University (17YC373) and the research startup foundation of China West Normal University(Grant No. 18Q060).

$凶 \quad$ Nan-jing Huang

nanjinghuang@hotmail.com

Da-peng Gao

gaodapeng126@126.com

1 School of Mathematics and Information, China West Normal University, Nanchong 637009, Sichuan, People's Republic of China

2 Department of Mathematics, Sichuan University, Chengdu 610064, Sichuan, People's Republic of China 


\section{Introduction}

One of the main goals in mathematical epidemiology is to understand how to control and eradicate infectious diseases. Meanwhile, diseases controlling can benefit from well understanding for dynamic behaviors of infectious diseases. To understand the mechanism of diseases transmission, researchers have studied many epidemic models (see [1-3,5-10,1215,17-23] and the references therein). For example, Guo et al. [8] constructed an SIR model to show the impact of discontinuous treatment on disease dynamics. Under certain conditions on the discontinuous treatment function, they determined the basic reproduction number $R_{0}$, confirmed the well-posedness of the model, described the structure of possible equilibria as well as established the stability/instability of the equilibria. After then, there have been several researches focusing on global dynamics of epidemic models with discontinuous treatment (see, for example, [22,23]).

Note that there are several infectious diseases (e.g. TB, HIV/AIDS, malaria, SARS etc.) which have latent periods (see, for example, $[17,18,23]$ ). Thus, it is necessary to consider models taking the latent periods into account. Recently, Zhang et al. [23] studied an SEIR epidemic model with a discontinuous treatment function. They obtained the basic reproductive number $R_{0}$, which is proved to be a sharp threshold value, completely determines the dynamics of the model. We note that a mathematical epidemic model can be characterized by the function describing the mechanism of transmission of the disease. This function is called incidence rate and depends generally on both the susceptible and infective classes, i.e., it takes the form $f(S, I)$, where $S$ is the number of susceptible and $I$ is the number of infective. Recently, many authors have studied several epidemic models with specific incidence rates (see, for example, [2,3,5-10,12-23] and the references therein). More precisely, Yang and Sun [22] considered a 2-dimensional SIR model involving a class of nonlinear incidence rate $S g(I)$ and a discontinuous treatment function. They showed that the global dynamics are fully determined by the basic reproduction number $R_{0}$, under certain conditions on the incidence rate and treatment function.

However, to the best of our knowledge, the study of the global stability for SEIR epidemic models with nonlinear incidence rates and discontinuous treatment functions is an untreated topic in the literature. Therefore, it would be important and interesting to study the global stability for an SEIR model with a nonlinear incidence rate and a discontinuous treatment function under mild conditions.

The remainder of this paper is organized as follows. In Sect. 2, we formulate a model and give some elementary assumptions on nonlinear incidence rates and discontinuous treatment functions. We also give the positivity and boundedness of the solution of the model in the sense of Filippov. In Sect. 3, we obtain the existence of possible equilibria, the basic reproduction number $R_{0}$, and the stability of equilibria. In Sect. 4 , the finite time convergence of the disease-free equilibria is discussed. Some numerical simulations, aimed at justifying the theoretical analysis, are reported in Sect. 5. The paper ends with conclusions in Sect. 6.

\section{Model formulation and preliminaries}

To derive our model, we split the total population into susceptible, exposed, infected and recovered classes, and denote the sizes by $S, E, I$ and $R$, respectively. The infected individuals will be given a discontinuous treatment. 
The above hypotheses lead to the following SEIR model with a class of nonlinear incidence rate $S(t) g(I(t))$ and a discontinuous treatment function $h(I(t))$ :

$$
\left\{\begin{array}{l}
\frac{d S}{d t}=\Lambda-\mu S-S g(I), \\
\frac{d E}{d t}=S g(I)-(\mu+\varepsilon) E, \\
\frac{d I}{d t}=\varepsilon E-(\mu+\alpha+\gamma) I-h(I), \\
\frac{d R}{d t}=\gamma I+h(I)-\mu R,
\end{array}\right.
$$

where $\Lambda$ is the growth rate of population, $\mu$ is the natural mortality rate, $\alpha$ is the death rate induced by disease, $\varepsilon$ and $\gamma$ are the rates of naturally leaving the exposed stage and infected stage, respectively. The function $h(I)=\varphi(I) I$ denotes the treatment rate. To define the basic reproduction number $R_{0}$ and indicate the existence of equilibriums, we furthermore give the following hypotheses.

(A1) $h(I)=\varphi(I) I$, where $\varphi: \mathbf{R}_{+} \rightarrow \mathbf{R}_{+}$is piecewise continuous and monotone nondecreasing, that is, $\varphi$ is continuous apart from a countable number of isolated points $\left\{\xi_{k}\right\}$, $\varphi\left(\xi_{k}^{+}\right)>\varphi\left(\xi_{k}^{-}\right)$holds, where $\varphi\left(\xi_{k}^{-}\right)$and $\varphi\left(\xi_{k}^{+}\right)$represent the left and right limits of $\varphi$ at $\left\{\xi_{k}\right\}$, respectively, and has only finite number of discontinuous points in any compact subset of $\mathbf{R}_{+}$. We assume that $\varphi$ is continuous for $I=0$.

(A2) $g(0)=0, g^{\prime}(I)>0$ and $g^{\prime \prime}(I) \leq 0$ for $I \geq 0$. Furthermore, we assume that the function $\phi(I)=\frac{g(I)}{I}$ is bounded.

Remark 2.1 (1) Clearly, (A2) shows that $\phi(I)=\frac{g(I)}{I}$ is a monotone decreasing function on $I>0$.

(2) It follows from the assumptions that system (2.1) always has a disease-free equilibrium point $P_{0}=\left(\frac{\Lambda}{\mu}, 0,0,0\right)$.

We always assume that (A1) and (A2) hold in the rest of this article unless a specific exception is made. Since the variable $R$ does not appear in the first three equations of model (2.1), we only need to study the first three equations of model (2.1), thereby lowering the order of the system to be studied, i.e.,

$$
\left\{\begin{array}{l}
\frac{d S}{d t}=\Lambda-\mu S-S g(I), \\
\frac{d E}{d t}=S g(I)-(\mu+\varepsilon) E, \\
\frac{d I}{d t}=\varepsilon E-(\mu+\alpha+\gamma) I-h(I) .
\end{array}\right.
$$

By the definition of the Filippov solution for differential equations with discontinuous righthand sides in $[4,11]$, we call that $(S(t), E(t), I(t))$ is a solution of model $(2.2)$ on $[0, T)$ $(0<T \leq \infty)$ with initial condition

$$
(S(0), E(0), I(0))=\left(S_{0}, E_{0}, I_{0}\right), \quad S_{0}, E_{0}, I_{0} \geq 0
$$


if it is absolutely continuous on any compact subinterval of $[0, T)$, and almost everywhere on $[0, T)$ (abbreviated to 'a.e. on $[0, T)$ ') satisfies the following differential inclusion:

$$
\left\{\begin{array}{l}
\frac{d S}{d t}=\Lambda-\mu S-S g(I), \\
\frac{d E}{d t}=S g(I)-(\mu+\varepsilon) E, \\
\frac{d I}{d t} \in \varepsilon E-(\mu+\alpha+\gamma) I-\overline{c o}[h(I)],
\end{array}\right.
$$

where $\overline{c o}[h(I)]=[h(I-0), h(I+0)]$. Here, $h(I-0)$ and $h(I+0)$ denote the left limit and the right limit of the function $h(I)$ at $I$, respectively.

From (A1), it is clear that the map

$$
(S, E, I) \longmapsto(\Lambda-\mu S-S g(I), S g(I)-(\mu+\varepsilon) E, \varepsilon E-(\mu+\alpha+\gamma) I-\overline{c o}[h(I)])
$$

is an upper semi-continuous set-valued map with non-empty compact convex values. By the measurable selection theorem ( [11], p.17, Theorem 2.2.5), if $(S(t), E(t), I(t))$ is a solution of model (2.2) on $[0, T)$, then there is a measurable function $m(t) \in \overline{c o}[h(I)]$ such that

$$
\left\{\begin{array}{l}
\frac{d S}{d t}=\Lambda-\mu S-S g(I), \\
\frac{d E}{d t}=S g(I)-(\mu+\varepsilon) E, \quad \text { a.e. on }[0, \mathrm{~T}) . \\
\frac{d I}{d t}=\varepsilon E-(\mu+\alpha+\gamma) I-m(t),
\end{array}\right.
$$

For biological reasons, we need to prove the positiveness and boundness of the solutions of model (2.2) with positive initial values.

Theorem 2.1 Let $(S(t), E(t), I(t))$ be the solution with initial condition (2.3) of model (2.2) on $[0, T)$. Then $(S(t), E(t), I(t))$ is nonnegative and bounded on $[0, T)$.

Proof Since the proof is similar to [23], we omit it here.

\section{Stability of equilibria of (2.2)}

In this section, we analyze model (2.2) by finding its equilibria and studying its stability.

An equilibrium of model (2.2) is, by definition, a constant solution of (2.2), i.e., $(S(t), E(t), I(t))=\left(S^{*}, E^{*}, I^{*}\right)$ for all $t \in[0,+\infty)$. Clearly, $\left(S^{*}, E^{*}, I^{*}\right)$ is an equilibrium of model (2.2) if and only if

$$
\left\{\begin{array}{l}
0=\Lambda-\mu S^{*}-S^{*} g\left(I^{*}\right) \\
0=S^{*} g\left(I^{*}\right)-(\mu+\varepsilon) E^{*} \\
0 \in \varepsilon E^{*}-(\mu+\alpha+\gamma) I^{*}-\overline{c o}\left[h\left(I^{*}\right)\right] .
\end{array}\right.
$$

By the first and second equations of (3.1), we conclude that

$$
S^{*}=\frac{\Lambda}{\mu+g\left(I^{*}\right)}, \quad E^{*}=\frac{\Lambda g\left(I^{*}\right)}{(\mu+\varepsilon)\left(\mu+g\left(I^{*}\right)\right)} .
$$

Substituting (3.2) into the third inclusion of (3.1) yields

$$
\frac{\Lambda \varepsilon g\left(I^{*}\right)}{(\mu+\varepsilon)\left[\mu+g\left(I^{*}\right)\right] I^{*}}-(\mu+\alpha+\gamma) \in \overline{c o}\left[\varphi\left(I^{*}\right)\right]
$$




$$
=\left[\varphi\left(I^{*}-0\right), \varphi\left(I^{*}+0\right)\right] .
$$

Let

$$
L(I)=\frac{\Lambda \varepsilon g(I)}{(\mu+\varepsilon)[\mu+g(I)] I}-(\mu+\alpha+\gamma)
$$

and

$$
R_{0}=\frac{\Lambda \varepsilon g^{\prime}(0)}{\mu(\mu+\varepsilon)(\mu+\alpha+\gamma+\varphi(0))} .
$$

We next claim that $R_{0}$ is the basic reproduction number which will determine the existence of an endemic equilibrium.

Theorem 3.1 If $R_{0}>1$, then there exists a unique endemic equilibrium $P^{*}$.

Proof If $R_{0}>1$, then

$$
\Lambda \varepsilon g^{\prime}(0)>\mu(\mu+\varepsilon)(\mu+\alpha+\gamma+\varphi(0))
$$

and so

$$
L\left(0^{+}\right)=\frac{\Lambda \varepsilon g^{\prime}(0)}{\mu(\mu+\varepsilon)}-(\mu+\alpha+\gamma)>\varphi(0) \geq 0 .
$$

This shows that the set

$$
\Gamma=\left\{I \mid L(I) \geq \varphi\left(I^{+}\right), I>0\right\}
$$

is nonempty. Further, a direct calculation gives

$$
\begin{aligned}
L^{\prime}(I) & =\frac{\Lambda \varepsilon\left[\mu g^{\prime}(I) I-\mu g(I)-g^{2}(I)\right]}{(\mu+\varepsilon)[\mu+g(I)]^{2} I^{2}} \\
& =\frac{\Lambda \varepsilon\left[g^{\prime}(I) \mu I-\mu g^{\prime}(\xi) I-g^{2}(I)\right]}{(\mu+\varepsilon)[\mu+g(I)]^{2} I^{2}},
\end{aligned}
$$

where $\xi \in(0, I)$. Noting $g(0)=0$ and $g^{\prime \prime}(I) \leq 0$ for $I>0$, we have $L^{\prime}(I)<0$. This shows that $L(I)$ is monotonously decreasing on $I>0$. Since $\varphi(I)$ is nondecreasing for $I>0$ and $L(I) \leq 0$ for

$$
I \geq \frac{\Lambda \varepsilon g(I)}{(\mu+\varepsilon)[\mu+g(I)](\mu+\alpha+\gamma)},
$$

we know that the set

$$
\Gamma=\left\{I \mid L(I) \geq \varphi\left(I^{+}\right), I>0\right\}
$$

is bounded. Let

$$
I^{*}=\sup \left\{I \mid L(I) \geq \varphi\left(I^{+}\right), I>0\right\} .
$$

Then $L\left(I^{*}\right) \geq \varphi\left(I^{*-}\right)$ and

$$
0<I^{*} \leq \frac{\Lambda \varepsilon g(I)}{(\mu+\varepsilon)[\mu+g(I)](\mu+\alpha+\gamma)} .
$$

We claim that

$$
L\left(I^{*}\right) \in\left[\varphi\left(I^{*-}\right), \varphi\left(I^{*+}\right)\right] .
$$


Otherwise,

$$
L\left(I^{*}\right)>\varphi\left(I^{*+}\right)=\lim _{I \rightarrow I^{*+}} \varphi(I) .
$$

It follows from (A1) that there exists a constant $\delta>0$ such that

$$
L\left(I^{*}+\delta\right)>\varphi\left(I^{*}+\delta\right)=\varphi\left(I^{*+}+\delta\right) .
$$

This contradicts the definition of $I^{*}$. Thus, $L\left(I^{*}\right) \in\left[\varphi\left(I^{*-}\right), \varphi\left(I^{*+}\right)\right]$ and $I^{*}$ is a positive solution to $L(I) \in \overline{c o}[\varphi(I)]$.

We next show the uniqueness of $I^{*}$. If inclusion (3.3) has another positive solution $I^{* *}$, then there exist $\eta_{1} \in \overline{c o}\left[\varphi\left(I^{*}\right)\right]$ and $\eta_{2} \in \overline{c o}\left[\varphi\left(I^{* *}\right)\right]$ such that

$$
\frac{\Lambda \varepsilon g\left(I^{*}\right)}{(\mu+\varepsilon)\left[\mu+g\left(I^{*}\right)\right] I^{*}}-(\mu+\alpha+\gamma)-\eta_{1}=0
$$

and

$$
\frac{\Lambda \varepsilon g\left(I^{* *}\right)}{(\mu+\varepsilon)\left[\mu+g\left(I^{* *}\right)\right] I^{* *}}-(\mu+\alpha+\gamma)-\eta_{2}=0 .
$$

Putting $k_{1}=g\left(I^{*}\right)$ and $k_{2}=g\left(I^{* *}\right)$, we have

$$
\begin{aligned}
0= & (\mu+\alpha+\gamma)\left(I^{*}-I^{* *}\right)+\eta_{1}\left(I^{*}-I^{* *}\right)+I^{* *}\left(\eta_{1}-\eta_{2}\right)+\mu(\mu+\alpha+\gamma) \\
& \times\left[\frac{I^{*}}{k_{1}}-\frac{I^{* *}}{k_{2}}\right]+\mu\left[\frac{\eta_{1} I^{*}}{k_{1}}-\frac{\eta_{2} I^{* *}}{k_{2}}\right] \\
= & {\left[(\mu+\alpha+\gamma)+\eta_{1}+I^{* *} H_{1}+\mu(\mu+\alpha+\gamma) H_{2}+\mu H_{3}\right]\left(I^{*}-I^{* *}\right), }
\end{aligned}
$$

where

$$
H_{1}=\frac{\eta_{1}-\eta_{2}}{I^{*}-I^{* *}}, \quad H_{2}=\frac{\frac{I^{*}}{k_{1}}-\frac{I^{* *}}{k_{2}}}{I^{*}-I^{* *}}, \quad H_{3}=\frac{\frac{\eta_{1} I^{*}}{k_{1}}-\frac{\eta_{2} I^{* *}}{k_{2}}}{I^{*}-I^{* *}} .
$$

Note that $H_{1} \geq 0$ for the monotonicity of $\varphi(I)$ and $H_{2} \geq 0$ for the monotonicity of $\frac{x}{g(x)}$. Moreover, the monotonicity of $\frac{x}{g(x)}$ and $\varphi(I)$ shows that $H_{3} \geq 0$. These facts together force the righthand side of (3.4) to be nonzero, a contradiction. So the uniqueness of $I^{*}$ is proved, which finishes the proof.

What follow is the global stability of the disease-free equilibrium and the endemic equilibrium. The proof falls naturally into several steps. We first prove their local stability as follows.

Theorem 3.2 The disease-free equilibrium $P_{0}$ is locally asymptotically stable if $R_{0}<1$, and is unstable if $R_{0}>1$.

Proof The Jacobian matrix of model (2.2) at $P_{0}$ is

$$
J\left(P_{0}\right)=\left(\begin{array}{ccc}
-\mu & 0 & -\frac{\Lambda}{\mu} g^{\prime}(0) \\
0 & -(\mu+\varepsilon) & \frac{\Lambda}{\mu} g^{\prime}(0) \\
0 & \varepsilon & -(\mu+\alpha+\gamma)-\varphi(0)
\end{array}\right)
$$

and the characteristic equation of $J\left(P_{0}\right)$ is

$$
(\lambda+\mu)\left[\lambda^{2}+(\mu+\varepsilon+\mu+\alpha+\gamma+\varphi(0)) \lambda+\right.
$$




$$
\left.+(\mu+\varepsilon)(\mu+\alpha+\gamma+\varphi(0))-\varepsilon \frac{\Lambda}{\mu} g^{\prime}(0)\right]=0 .
$$

Clearly, equation (3.5) has three roots $\lambda_{1}=-\mu<0, \lambda_{2}$ and $\lambda_{3}$ satisfying

$$
\lambda_{2}+\lambda_{3}=-(\mu+\varepsilon+\mu+\alpha+\gamma+\varphi(0))<0
$$

and

$$
\lambda_{2} \lambda_{3}=(\mu+\varepsilon)(\mu+\alpha+\gamma+\varphi(0))\left(1-R_{0}\right) .
$$

It follows from (3.6) and (3.7) that both the real parts of $\lambda_{2}$ and $\lambda_{3}$ are negative when $R_{0}<1$.

When $R_{0}>1$, one of $\lambda_{2}$ and $\lambda_{3}$ is a number with a positive real part. Thus the diseasefree equilibrium $P_{0}$ is locally asymptotically stable if $R_{0}<1$ and unstable if $R_{0}>1$, which completes the proof.

Theorem 3.3 If $R_{0}>1$ and $\varphi$ is differential at $I^{*}$, then the endemic equilibrium $P^{*}$ is locally asymptotically stable.

Proof The Jacobian matrix of model (2.2) at the endemic equilibrium $P^{*}=\left(S^{*}, E^{*}, I^{*}\right)$ is

$$
J\left(P^{*}\right)=\left(\begin{array}{ccc}
-\mu-g\left(I^{*}\right) & 0 & -S^{*} g^{\prime}\left(I^{*}\right) \\
g\left(I^{*}\right) & -(\mu+\varepsilon) & S^{*} g^{\prime}\left(I^{*}\right) \\
0 & \varepsilon & -(\mu+\alpha+\gamma)-\varphi\left(I^{*}\right)-\varphi^{\prime}\left(I^{*}\right) I^{*}
\end{array}\right) .
$$

Replacing $-(\mu+\alpha+\gamma)-\varphi\left(I^{*}\right)$ by $-\frac{\Lambda \varepsilon g\left(I^{*}\right)}{(\mu+\varepsilon)\left[\mu+g\left(I^{*}\right)\right] I^{*}}$ gives

$$
J\left(P^{*}\right)=\left(\begin{array}{ccc}
-\mu-g\left(I^{*}\right) & 0 & -S^{*} g^{\prime}\left(I^{*}\right) \\
g\left(I^{*}\right) & -(\mu+\varepsilon) & S^{*} g^{\prime}\left(I^{*}\right) \\
0 & \varepsilon & -\frac{\Lambda \varepsilon g\left(I^{*}\right)}{(\mu+\varepsilon)\left[\mu+g\left(I^{*}\right)\right] I^{*}}-\varphi^{\prime}\left(I^{*}\right) I^{*}
\end{array}\right) .
$$

The characteristic equation of $J\left(P^{*}\right)$ is

$$
\lambda^{3}+a_{1} \lambda^{2}+a_{2} \lambda+a_{3}=0,
$$

where

$$
\begin{aligned}
a_{1}= & \mu+g\left(I^{*}\right)+\mu+\varepsilon+\frac{\Lambda \varepsilon g\left(I^{*}\right)}{(\mu+\varepsilon)\left[\mu+g\left(I^{*}\right)\right] I^{*}}+\varphi^{\prime}\left(I^{*}\right) I^{*}>0, \\
a_{2}= & \left(\mu+g\left(I^{*}\right)\right)(\mu+\varepsilon)+\left(\mu+g\left(I^{*}\right)\right)\left(\frac{\Lambda \varepsilon g\left(I^{*}\right)}{(\mu+\varepsilon)\left[\mu+g\left(I^{*}\right)\right] I^{*}}+\varphi^{\prime}\left(I^{*}\right) I^{*}\right) \\
& +(\mu+\varepsilon)\left(\frac{\Lambda \varepsilon g\left(I^{*}\right)}{(\mu+\varepsilon)\left[\mu+g\left(I^{*}\right)\right] I^{*}}+\varphi^{\prime}\left(I^{*}\right) I^{*}\right)-\frac{\Lambda \varepsilon g^{\prime}\left(I^{*}\right)}{\mu+g\left(I^{*}\right)} \\
> & (\mu+\varepsilon) \frac{\Lambda \varepsilon g\left(I^{*}\right)}{(\mu+\varepsilon)\left[\mu+g\left(I^{*}\right)\right] I^{*}}-\frac{\Lambda \varepsilon g^{\prime}\left(I^{*}\right)}{\mu+g\left(I^{*}\right)} \\
= & \frac{\varepsilon \Lambda g^{\prime}(\xi)-\varepsilon \Lambda g^{\prime}\left(I^{*}\right)}{\mu+g\left(I^{*}\right)} \\
> & 0
\end{aligned}
$$

for some $\xi \in\left(0, I^{*}\right)$, and

$$
a_{3}=\left(\mu+g\left(I^{*}\right)\right)(\mu+\varepsilon)\left(\frac{\varepsilon \Lambda g\left(I^{*}\right)}{(\mu+\varepsilon)\left[\mu+g\left(I^{*}\right)\right] I^{*}}+\varphi^{\prime}\left(I^{*}\right) I^{*}\right)-\mu \varepsilon \frac{\Lambda g^{\prime}\left(I^{*}\right)}{\mu+g\left(I^{*}\right)}
$$




$$
\begin{aligned}
& >\frac{\mu \varepsilon \Lambda g\left(I^{*}\right)}{\left[\mu+g\left(I^{*}\right)\right] I^{*}}-\frac{\mu \varepsilon \Lambda g^{\prime}\left(I^{*}\right)}{\mu+g\left(I^{*}\right)} \\
& =\frac{\mu \varepsilon \Lambda\left[g^{\prime}(\eta)-g^{\prime}\left(I^{*}\right)\right]}{\mu+g\left(I^{*}\right)} \\
& >0
\end{aligned}
$$

for some $\eta \in\left(0, I^{*}\right)$. Now it follows that

$$
\begin{aligned}
& a_{1} a_{2}-a_{3} \\
&=\quad\left[\mu+g\left(I^{*}\right)+\mu+\varepsilon+\frac{\varepsilon \Lambda g\left(I^{*}\right)}{(\mu+\varepsilon)\left[\mu+g\left(I^{*}\right)\right] I^{*}}+\varphi^{\prime}\left(I^{*}\right) I^{*}\right] \times \\
&\left\{\left(\mu+g\left(I^{*}\right)\right)(\mu+\varepsilon)+\left(\mu+g\left(I^{*}\right)\right)\left(\frac{\varepsilon \Lambda g\left(I^{*}\right)}{(\mu+\varepsilon)\left[\mu+g\left(I^{*}\right)\right] I^{*}}+\varphi^{\prime}\left(I^{*}\right) I^{*}\right)\right. \\
&\left.+(\mu+\varepsilon)\left(\frac{\varepsilon \Lambda g\left(I^{*}\right)}{(\mu+\varepsilon)\left[\mu+g\left(I^{*}\right)\right] I^{*}}+\varphi^{\prime}\left(I^{*}\right) I^{*}\right)-\frac{\varepsilon \Lambda g^{\prime}\left(I^{*}\right)}{\mu+g\left(I^{*}\right)}\right\} \\
&-\left\{\left(\mu+g\left(I^{*}\right)\right)(\mu+\varepsilon)\left(\frac{\varepsilon \Lambda g\left(I^{*}\right)}{(\mu+\varepsilon)\left[\mu+g\left(I^{*}\right)\right] I^{*}}+\varphi^{\prime}\left(I^{*}\right) I^{*}\right)-\frac{\mu \varepsilon \Lambda g^{\prime}\left(I^{*}\right)}{\mu+g\left(I^{*}\right)}\right\} \\
&>\left(\mu+g\left(I^{*}\right)\right)(\mu+\varepsilon)\left(\frac{\varepsilon \Lambda g\left(I^{*}\right)}{(\mu+\varepsilon)\left[\mu+g\left(I^{*}\right)\right] I^{*}}+\varphi^{\prime}\left(I^{*}\right) I^{*}\right) \\
&+\left\{\frac{\mu(\mu+\varepsilon) \varepsilon \Lambda g\left(I^{*}\right)}{(\mu+\varepsilon)\left[\mu+g\left(I^{*}\right)\right] I^{*}}-\frac{\mu \varepsilon \Lambda g^{\prime}\left(I^{*}\right)}{\mu+g\left(I^{*}\right)}\right\} \\
&-\left(\frac{\left(\mu+g\left(I^{*}\right)\right)(\mu+\varepsilon) \varepsilon \Lambda g\left(I^{*}\right)}{(\mu+\varepsilon)\left[\mu+g\left(I^{*}\right)\right] I^{*}}+\left(\mu+g\left(I^{*}\right)\right)(\mu+\varepsilon) \varphi^{\prime}\left(I^{*}\right) I^{*}\right) \\
&+\frac{\mu \varepsilon \Lambda g^{\prime}\left(I^{*}\right)}{\mu+g\left(I^{*}\right)} \\
&= \frac{\mu \varepsilon \Lambda g\left(I^{*}\right)}{\left[\mu+g\left(I^{*}\right)\right] I^{*}} \\
&> 0 .
\end{aligned}
$$

Therefore, all of the assumptions of the Routh-Hurwitz criteria are satisfied. Thus it follows that the endemic equilibrium $P^{*}$ of (2.2), which exists if $R_{0}>1$, is always locally asymptotically stable. The proof is completed.

We next discuss the global stability of the disease-free equilibrium $P_{0}$ and endemic equilibrium $P^{*}$. To this end, we need to apply the LaSalle-type invariance principle for the differential inclusion (Theorem 3 in [1]) to prove the global stability.

Theorem 3.4 If $R_{0} \leq 1$, then the disease-free equilibrium $P_{0}$ of (2.4) is globally asymptotically stable.

Proof We move $P_{0}$ to the origin firstly by setting $x=S-\frac{\Lambda}{\mu}$. Then, (2.4) is transformed to the following form:

$$
\left\{\begin{array}{l}
\frac{d x}{d t}=-\mu x-x g(I)-\frac{\Lambda}{\mu} g(I), \\
\frac{d E}{d t}=x g(I)+\frac{\Lambda}{\mu} g(I)-(\mu+\varepsilon) E, \\
\frac{d I}{d t} \in \varepsilon E-(\mu+\alpha+\gamma) I-\overline{c o}[\varphi(I)] I .
\end{array}\right.
$$


Consider the Lyapunov function

$$
V_{1}(x, E, I)=\frac{x^{2}}{2}+\frac{\Lambda}{\mu} E+\frac{\Lambda}{\mu} \frac{\mu+\varepsilon}{\varepsilon} I .
$$

Let

$$
\mathbf{G}(x, E, I)=\left(\begin{array}{c}
-\mu x-x g(I)-\frac{\Lambda}{\mu} g(I) \\
x g(I)+\frac{\Lambda}{\mu} g(I)-(\mu+\varepsilon) E \\
\varepsilon E-(\mu+\alpha+\gamma) I-\overline{c o}[\varphi(I)] I
\end{array}\right) .
$$

Then, for any $\mathbf{V}=\left(v_{1}, v_{2}, v_{3}\right)^{T} \in \mathbf{G}(x, E, I)$, there exists an $\eta(t) \in \overline{c o}[\varphi(I)]$ such that

$$
\mathbf{V}=\left(\begin{array}{c}
-\mu x-x g(I)-\frac{\Lambda}{\mu} g(I) \\
x g(I)+\frac{\Lambda}{\mu} g(I)-(\mu+\varepsilon) E \\
\varepsilon E-(\mu+\alpha+\gamma) I-\eta(t) I
\end{array}\right)
$$

and so

$$
\begin{aligned}
& \left\langle\nabla V_{1}(x, E, I), \mathbf{V}\right\rangle \\
& =\left(x, \frac{\Lambda}{\mu}, \frac{\Lambda}{\mu} \frac{\mu+\varepsilon}{\varepsilon}\right) \times\left(\begin{array}{c}
-\mu x-x g(I)-\frac{\Lambda}{\mu} g(I) \\
x g(I)+\frac{\Lambda}{\mu} g(I)-(\mu+\varepsilon) E \\
\varepsilon E-(\mu+\alpha+\gamma) I-\eta(t) I
\end{array}\right) \\
& =-\mu x^{2}-x^{2} g(I)+\frac{I \Lambda(\mu+\varepsilon)}{\mu \varepsilon}\left\{\frac{\varepsilon \Lambda g(I)}{\mu I(\mu+\varepsilon)}-[\mu+\alpha+\gamma+\eta(t)]\right\} .
\end{aligned}
$$

It follows from Remark 2.1 that

$$
\frac{g(I)}{I} \leq \lim _{I \rightarrow 0^{+}} \frac{g(I)}{I}=g^{\prime}(0) .
$$

Using the fact $\eta(t) \geq \varphi(0)$, we have

$$
\left\langle\nabla V_{1}, \mathbf{V}\right\rangle \leq-\mu x^{2}-x^{2} g(I)+\frac{\Lambda(\mu+\varepsilon)}{\mu \varepsilon}\left\{\frac{\varepsilon \Lambda}{\mu(\mu+\varepsilon)} g^{\prime}(0)-[\mu+\alpha+\gamma+\varphi(0)]\right\} I .
$$

Since $R_{0} \leq 1$, we know that $\left\langle\nabla V_{1}, \mathbf{V}\right\rangle \leq 0$.

Furthermore, when $R_{0}<1$,

$$
\begin{aligned}
Z_{V_{1}} & :=\left\{(x, E, I) \in R^{3} \mid\left\langle\nabla V_{1}(x, E, I), \mathbf{V}\right\rangle=0, \mathbf{V} \in \mathbf{G}(x, E, I)\right\} \\
& =\{(0, E, 0) \mid E>0\} .
\end{aligned}
$$

This shows that $I=0$ and so $\frac{d E}{d t}=-(\mu+\varepsilon) E$. It is easy to see that $\lim _{t \rightarrow \infty} E(t)=0$. For any $l>0$, we set

$$
V_{1}^{l}=\left\{(x, E, I) \in R^{3} \mid V_{1}(x, E, I) \leq l\right\} .
$$

Then the largest weakly invariant subset of $\overline{Z_{V_{1}}} \cap V_{1}^{l}$ is the singleton $M=\{(0,0,0)\}$.

When $R_{0}=1$, we have

$$
Z_{V_{1}}=\{(0, E, 0) \mid E \geq 0\} \bigcup\{(0, E, I) \mid \eta(t)=\varphi(0), I>0\} .
$$


From the first equation of (3.8) and $x=0$, we can conclude that $I=0$. Thus, the largest weakly invariant subset of $\overline{Z_{V_{1}}} \cap V_{1}^{l}$ is also the singleton $M=\{(0,0,0)\}$, which leads to globally asymptotical stability of $(0,0,0)$ due to the invariance theorem, and thus $P_{0}$ is globally asymptotically stable. The proof is completed.

The following theorem deals with the globally asymptotic stability of the endemic equilibrium $P^{*}$ when $R_{0}>1$.

Theorem 3.5 If $R_{0}>1$, then the unique endemic equilibrium $P^{*}$ of (2.4) is globally asymptotically stable.

Proof Consider

$$
\begin{aligned}
V_{2}(S, E, I)= & \left(S-S^{*}-S^{*} \ln \frac{S}{S^{*}}\right)+\left(E-E^{*}-E^{*} \ln \frac{E}{E^{*}}\right) \\
& +\frac{\mu+\varepsilon}{\varepsilon}\left(I-I^{*}-I^{*} \ln \frac{I}{I^{*}}\right) .
\end{aligned}
$$

Let

$$
\eta^{*}=\frac{1}{I^{*}}\left(\varepsilon E^{*}-(\mu+\alpha+\gamma) I^{*}\right) \in \overline{c o}\left[\varphi\left(I^{*}\right)\right]
$$

and

$$
\mathbf{H}(S, E, I)=\left(\begin{array}{c}
\Lambda-\mu S-S g(I) \\
S g(I)-(\mu+\varepsilon) E \\
\varepsilon E-(\mu+\alpha+\gamma) I-\overline{c o}[\varphi(I)] I
\end{array}\right)
$$

For any $\mathbf{V}=\left(v_{1}, v_{2}, v_{3}\right)^{T} \in \mathbf{H}(S, E, I)$, there exists an $\eta(t) \in \overline{c o}[\varphi(I)]$ such that

$$
\mathbf{V}=\left(\begin{array}{c}
\Lambda-\mu S-S g(I) \\
S g(I)-(\mu+\varepsilon) E \\
\varepsilon E-(\mu+\alpha+\gamma) I-\eta(t) I
\end{array}\right) .
$$

Thus, it follows that

$$
\begin{aligned}
\langle\nabla & \left.V_{2}(S, E, I), \mathbf{V}\right\rangle \\
= & \left(1-\frac{S^{*}}{S}, 1-\frac{E^{*}}{E}, \frac{\mu+\varepsilon}{\varepsilon}\left(1-\frac{I^{*}}{I}\right)\right) \times\left(\begin{array}{c}
\Lambda-\mu S-S g(I) \\
S g(I)-(\mu+\varepsilon) E \\
\varepsilon E-(\mu+\alpha+\gamma) I-\eta(t) I
\end{array}\right) \\
= & \Lambda-\mu S-\frac{\Lambda}{S} S^{*}+\mu S^{*}+S^{*} g(I)-S g(I) \frac{E^{*}}{E}+(\mu+\varepsilon) E^{*}-(\mu+\varepsilon) \frac{E I^{*}}{I} \\
& -\frac{(\mu+\varepsilon)(\mu+\alpha+\gamma+\eta(t))}{\varepsilon}\left(I-I^{*}\right) \\
= & 3 S^{*} g\left(I^{*}\right)+\mu S^{*}-\mu S-\frac{\mu S^{* 2}}{S}-\frac{S^{* 2} g\left(I^{*}\right)}{S}+\mu S^{*}+S^{*} g(I) \\
& +\frac{\mu+\varepsilon}{\varepsilon}\left(\varepsilon \frac{E^{*}}{I^{*}}-(\mu+\alpha+\gamma+\eta(t))\left(I-I^{*}\right)\right)-S g(I) \frac{E^{*}}{E} \\
& -\frac{S^{*} g\left(I^{*}\right) E I^{*}}{E^{*} I}-\frac{S^{*} g\left(I^{*}\right) I}{I^{*}} \\
= & -S^{*} g\left(I^{*}\right)-\frac{S^{*} g\left(I^{*}\right) I}{I^{*}}+S^{*} g(I)+\frac{S^{*} g^{2}\left(I^{*}\right) I}{g(I) I^{*}}
\end{aligned}
$$




$$
\begin{aligned}
& +\frac{\mu+\varepsilon}{\varepsilon}\left(\varepsilon \frac{E^{*}}{I^{*}}-(\mu+\alpha+\gamma+\eta(t))\right)\left(I-I^{*}\right)+4 S^{*} g\left(I^{*}\right)-\frac{S^{* 2} g\left(I^{*}\right)}{S} \\
& -\frac{S g(I) E^{*}}{E}-\frac{S^{*} g^{2}\left(I^{*}\right) I}{g(I) I^{*}}-\frac{S^{*} g\left(I^{*}\right) E I^{*}}{I E^{*}}+2 \mu S^{*}-\mu S-\frac{\mu S^{* 2}}{S} \\
= & S^{*} g\left(I^{*}\right)\left[-1-\frac{I}{I^{*}}+\frac{g(I)}{g\left(I^{*}\right)}+\frac{g\left(I^{*}\right) I}{g(I) I^{*}}\right]+\frac{\mu+\varepsilon}{\varepsilon}\left(\eta^{*}-\eta\right)\left(I-I^{*}\right) \\
& +S^{*} g\left(I^{*}\right)\left[4-\frac{S^{*}}{S}-\frac{S g(I) E^{*}}{S^{*} g\left(I^{*}\right) E}-\frac{g\left(I^{*}\right) I}{g(I) I^{*}}-\frac{E I^{*}}{I E^{*}}\right]+\mu S^{*}\left(2-\frac{S}{S^{*}}-\frac{S^{*}}{S}\right) \\
= & S^{*} g\left(I^{*}\right)\left[\frac{I}{I^{*}}-\frac{g(I)}{g\left(I^{*}\right)}\right]\left[\frac{g\left(I^{*}\right)}{g(I)}-1\right]+\frac{\mu+\varepsilon}{\varepsilon}\left(\eta^{*}-\eta\right)\left(I-I^{*}\right) \\
& +S^{*} g\left(I^{*}\right)\left[4-\frac{S^{*}}{S}-\frac{S g(I) E^{*}}{S^{*} g\left(I^{*}\right) E}-\frac{g\left(I^{*}\right) I}{g(I) I^{*}}-\frac{E I^{*}}{I E^{*}}\right] \\
& +\mu S^{*}\left(1-\frac{S}{S^{*}}\right)\left(1-\frac{S^{*}}{S}\right) .
\end{aligned}
$$

One can see that $\left(1-\frac{S}{S^{*}}\right)\left(1-\frac{S^{*}}{S}\right) \leq 0$. Since the arithmetic mean is greater than or equal to the geometric mean, we have

$$
\frac{S^{*}}{S}+\frac{S g(I) E^{*}}{S^{*} g\left(I^{*}\right) E}+\frac{g\left(I^{*}\right) I}{g(I) I^{*}}+\frac{E I^{*}}{I E^{*}} \geq 4 .
$$

The monotonicity of $g(I)$ and $\phi(I)$ gives

$$
\frac{I}{I^{*}} \leq \frac{g(I)}{g\left(I^{*}\right)} \leq 1\left(0<I<I^{*}\right), \quad 1 \leq \frac{g(I)}{g\left(I^{*}\right)} \leq \frac{I}{I^{*}}\left(I \geq I^{*}\right) .
$$

Thus,

$$
\left[\frac{I}{I^{*}}-\frac{g(I)}{g\left(I^{*}\right)}\right]\left[\frac{g\left(I^{*}\right)}{g(I)}-1\right] \leq 0, \quad \forall I>0 .
$$

Since $\left(\eta^{*}-\eta\right)\left(I-I^{*}\right) \leq 0$ for the monotonicity of $\varphi$, we obtain

$$
\left\langle\nabla V_{2}(S, E, I), \mathbf{V}\right\rangle \leq 0
$$

Define

$$
\begin{aligned}
Z_{V_{2}} & :=\left\{(S, E, I) \in R_{+}^{3} \mid \exists \mathbf{V} \in \mathbf{H}(S, E, I),\left\langle\nabla V_{2}(S, E, I), \mathbf{V}\right\rangle=0\right\} \\
& =\left\{\left(S^{*}, E^{*}, I^{*}\right)\right\} \bigcup\left\{\left(S^{*}, E, I\right) \mid \frac{E}{E^{*}}=\frac{I}{I^{*}}=\frac{g(I)}{g\left(I^{*}\right)}, \eta^{*}=\eta(t)\right\} .
\end{aligned}
$$

It follows from $S=S^{*}$ and the first equation of (2.4) that $I=I^{*}$ and so $E=E^{*}$. Consequently, for any $l>0$, the largest weakly invariant subset of $\overline{Z_{V_{2}}} \cap V^{l}$ of (2.4) is the singleton $\left\{\left(S^{*}, E^{*}, I^{*}\right)\right\}$, where

$$
V^{l}=\left\{\left(S-S^{*}, E-E^{*}, I-I^{*}\right) \in R^{3} \mid V_{2}(S, E, I) \leq l\right\} .
$$

Therefore, $P^{*}$ is globally asymptotically stable if $R_{0}>1$. This ends the proof. 


\section{Global convergence in finite time}

A very meaningful and desirable situation is the global convergence to the disease-free equilibrium $P_{0}$ in finite time, which is an important feature contributed to distinguish discontinuous ODE systems and smooth ODE systems. In this section, we will show that the solutions converge to the disease-free equilibrium in finite time. From now on we make the assumption:

(A3) $h(I):[0,+\infty) \longrightarrow[0,+\infty)$ is nondecreasing and has at most a finite number of jump discontinuities in every compact interval. Moreover, $h(0)=0$ and $h(I)$ is discontinuous at $I=0$.

Under the assumption (A3), it is easy to see that $\left(\frac{\Lambda}{\mu}, 0,0\right)$ is disease-free equilibrium of (2.4). Let $x=S-\frac{\Lambda}{\mu}$. Then, (2.4) can be transformed to

$$
\left\{\begin{array}{l}
\frac{d x}{d t}=-\mu x-x g(I)-\frac{\Lambda}{\mu} g(I) \\
\frac{d E}{d t}=\left(x+\frac{\Lambda}{\mu}\right) g(I)-(\mu+\varepsilon) E \\
\frac{d I}{d t} \in \varepsilon E-(\mu+\alpha+\gamma) I-\overline{c o}[h(I)] .
\end{array}\right.
$$

From (2.5), there exists a measurable function $\eta(t) \in \overline{c o}[h(I)]$ corresponding to $(x(t), E(t), I(t))$ such that

$$
\left\{\begin{array}{l}
\frac{d x}{d t}=-\mu x-x g(I)-\frac{\Lambda}{\mu} g(I), \\
\frac{d E}{d t}=\left(x+\frac{\Lambda}{\mu}\right) g(I)-(\mu+\varepsilon) E, \text { for a.e. } t \in[0,+\infty) . \\
\frac{d I}{d t}=\varepsilon E-(\mu+\alpha+\gamma) I-\eta(t) .
\end{array}\right.
$$

Let $V_{1}(x, E, I)$ be the same Lyapunov function as in the proof of Theorem 3.4. Then, computing its derivative along the solutions of (4.2) gives

$$
\begin{aligned}
\frac{d V_{1}}{d t}= & x\left[-\mu x-x g(I)-\frac{\Lambda}{\mu} g(I)\right]+\frac{\Lambda}{\mu}\left[\left(x+\frac{\Lambda}{\mu}\right) g(I)-(\mu+\varepsilon) E\right] \\
& +\frac{\Lambda}{\mu} \frac{\mu+\varepsilon}{\varepsilon}[\varepsilon E-(\mu+\alpha+\gamma) I-\eta(t)] \\
= & -\mu x^{2}-x^{2} g(I)+\frac{\Lambda}{\mu}\left[\frac{\Lambda}{\mu} g(I)-\frac{(\mu+\varepsilon)(\mu+\alpha+\gamma)}{\varepsilon} I\right]-\frac{\Lambda(\mu+\varepsilon)}{\mu \varepsilon} \eta(t) .
\end{aligned}
$$

By (A3), we know that $\eta(t) \geq h\left(0^{+}\right)$. When $\frac{g(I)}{I} \leq \frac{\mu(\mu+\varepsilon)(\mu+\alpha+\gamma)}{\varepsilon \Lambda}$, we have

$$
\frac{d V_{1}}{d t} \leq-\frac{\Lambda}{\mu} \frac{\mu+\varepsilon}{\varepsilon} h\left(0^{+}\right) .
$$

Integrating both sides of inequality (4.3) from 0 to $t$, we have

$$
0 \leq V_{1}(x, E, I) \leq V_{1}(x(0), E(0), I(0))-\frac{\Lambda}{\mu} \frac{\mu+\varepsilon}{\varepsilon} h\left(0^{+}\right) t .
$$


This implies that $V_{1}(x, E, I)=0$ for $t>t^{*}$ which means $(x, E, I)=(0,0,0)$ and hence $(S(t), E(t), I(t))=\left(\frac{\Lambda}{\mu}, 0,0\right)$ for $t>t^{*}$, where

$$
t^{*}=\frac{\mu \varepsilon}{(\mu+\varepsilon) \Lambda h\left(0^{+}\right)}\left(\frac{\left(S(0)-\frac{\Lambda}{\mu}\right)^{2}}{2}+\frac{\Lambda}{\mu} E(0)+\frac{\Lambda}{\mu} \frac{\mu+\varepsilon}{\varepsilon} I(0)\right) .
$$

Summarizing the above analysis, we obtain the following theorem.

Theorem 4.1 Suppose that (A2) and (A3) hold. If $\frac{g(I)}{I} \leq \frac{\mu(\mu+\varepsilon)(\mu+\alpha+\gamma)}{\varepsilon \Lambda}$, then every solution of model (2.4) with initial condition $S(0)=S_{0} \geq 0, E(0)=E_{0} \geq 0$ and $I(0)=I_{0} \geq 0$ converges to $P_{0}$ in finite time, i.e., the disease goes to extinction in finite time. More precisely, $(S(t), E(t), I(t))=\left(\frac{\Lambda}{\mu}, 0,0\right)$ for $t>t^{*}$, where $t^{*}$ is defined in (4.5).

\section{Numerical examples}

In this section, four numerical examples of system (2.4) are presented to justify the theorems obtained in Sect. 3. All simulations are done using the function ode45, which is MATLAB's standard solver for ordinary differential equations (ODEs). This function implements a Runge-Kutta method with a variable time step for efficient computation. More details on this approach can be found in [16].

As the present study is not a case study, no real data are available. Hence, the choice of parametric values is hypothetical with appropriate units and does not base on data. They are chosen only for illustrative purpose. Because the parametric values are not related to a specific disease, system (2.4) can be considered to be dimensionless. The interval of time is supposed to be $[0,200]$, while the various set of initial size of population are assumed to be $(S(0), E(0), I(0))=(200,150,50)$. From the theoretical analysis in Sect. 3, we know that the stability of the disease-free equilibrium and the endemic equilibrium depend on the basic reproduction number $R_{0}$ which is reflected by the values of $\Lambda, \mu, \varepsilon, \alpha, \gamma, \varphi(0)$ and $g^{\prime}(0)$. We choose $\varphi(I)$ and $g(I)$ satisfying conditions $\left(A_{1}\right)$ and $\left(A_{2}\right)$, respectively. Here we present four numerical examples to discuss the effect of the choice of the parameters, treatment rate and nonlinear incidence rate on the basic reproduction number $R_{0}$, and to see the importance of the assumptions of Theorems 3.4 and 3.5.

Example 1 Consider system (2.4) with $\Lambda=100, \mu=0.1, \varepsilon=0.7, \alpha=0.2, \gamma=$ $0.5, S(0)=200, E(0)=150, I(0)=50$. Fix $g(I)=\frac{I}{I+10}$ and

$$
\varphi(I)=\left\{\begin{array}{lr}
I+100, & 0 \leq I<5 \\
110, & I \geq 5 .
\end{array}\right.
$$

It is easy to check that $R_{0}=0.8681$. It follows by Theorem 3.4 that system (2.4) has only a disease-free equilibrium, which is globally asymptotically stable. Figures 1, 2 and 3 show the corresponding time plot.

Example 2 Set $g(I)=\frac{I}{I+100}$ and

$$
\varphi(I)= \begin{cases}0.4, & I<20 \\ 0.5, & I \geq 20\end{cases}
$$


Fig. 1 Time series plot of the susceptible population for $\mathcal{R}_{0}=0.8681<1$ with initial conditions, parameter values as in Example 1

Fig. 2 Time series plot of the exposed population for

$\mathcal{R}_{0}=0.8681<1$ with initial conditions, parameter values as in Example 1

Fig. 3 Time series plot of the infective population for $\mathcal{R}_{0}=0.8681<1$ with initial conditions, parameter values as in Example 1
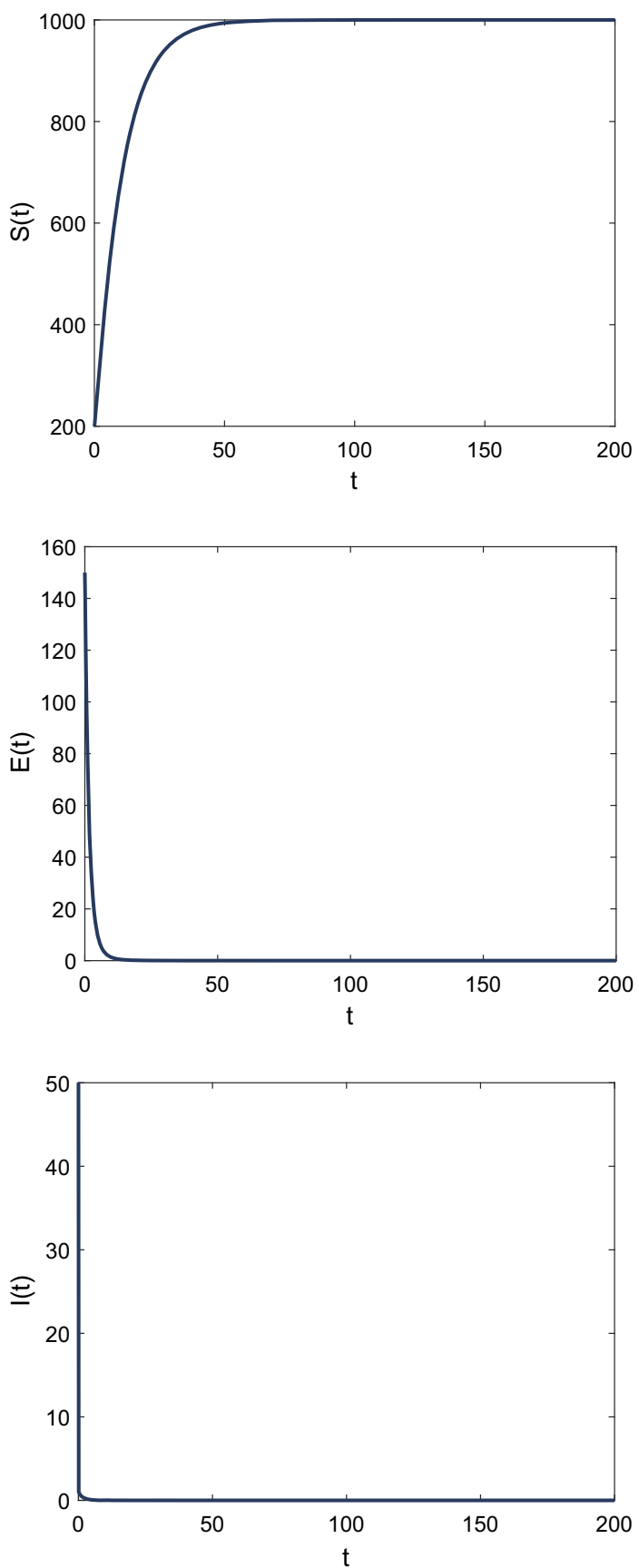
Fig. 4 Time series plot of the susceptible population for $\mathcal{R}_{0}=7.2917>1$ with initial conditions, parameter values as in Example 2

Fig. 5 Time series plot of the exposed population for $\mathcal{R}_{0}=7.2917>1$ with initial conditions, parameter values as in Example 2
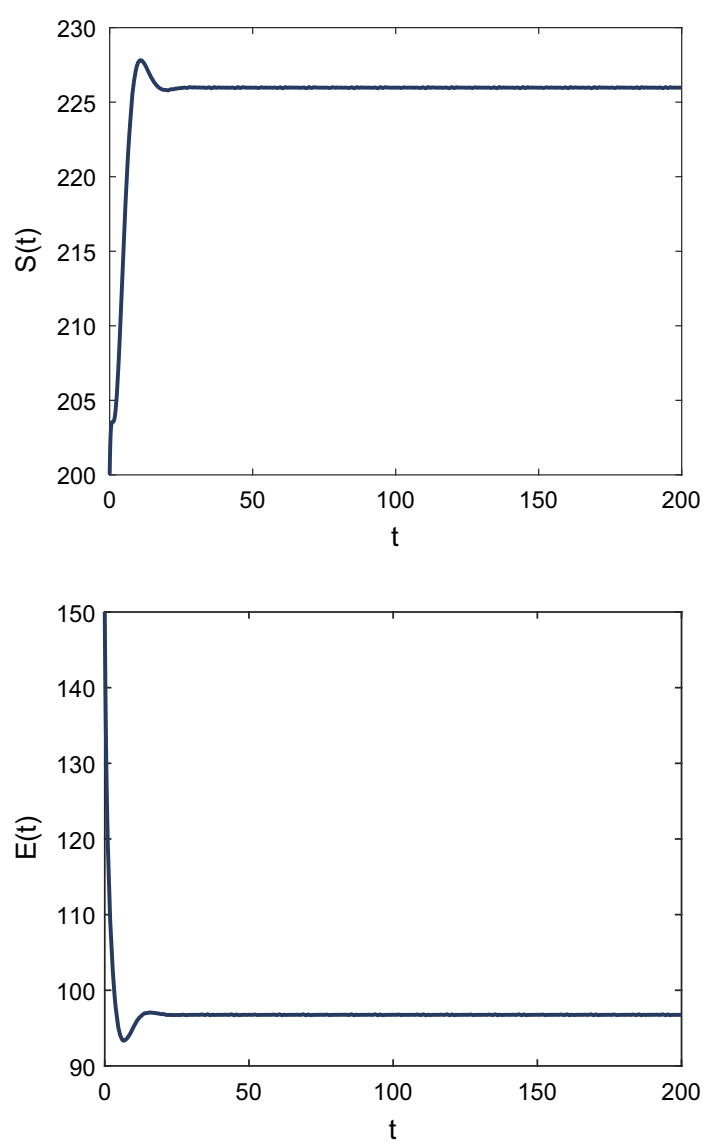

All values of other parameters are as in Example 1. It is easy to check that $R_{0}=7.2917$, which does not satisfy the assumptions of Theorem 3.4. Simulation results show that system (2.4) has no disease-free equilibrium, which is contrary to what Theorem 3.4 predicts. Figures 4, 5 and 6 show the corresponding time plot.

Example 3 Consider system (2.4) with $\Lambda=300, \mu=0.1, \varepsilon=0.7, \alpha=0.2, \gamma=$ $0.5, S(0)=200, E(0)=150, I(0)=50 . g(I)$ and $\varphi(I)$ are the same as in Example 1 . It is easy to check that $R_{0}=2.6042$. It follows by Theorem 3.5 that system (2.4) has a unique endemic equilibrium, which is globally asymptotically stable. Figures 7, 8 and 9 show the corresponding time plot.

Example 4 Take $g(I)=\frac{I}{I+1000}$. All of the other parameters and function are as in Example 2 . It is easy to check that $R_{0}=0.7292$, which does not satisfy the assumptions of Theorem 3.5. Simulation results show that system (2.4) has a disease-free equilibrium, which is contrary to what Theorem 3.5 predicts. Figures 10,11 and 12 show the corresponding time plot.

Remark 5.1 Examples 1-4 indicate that the basic reproductive number $R_{0}$ is critical in determining the dynamical behaviors of system (2.4). 
Fig. 6 Time series plot of the infective population for $\mathcal{R}_{0}=7.2917>1$ with initial conditions, parameter values as in Example 2

Fig. 7 Time series plot of the susceptible population for $\mathcal{R}_{0}=2.6042>1$ with initial conditions, parameter values as in Example 3

Fig. 8 Time series plot of the exposed population for $\mathcal{R}_{0}=2.6042>1$ with initial conditions, parameter values as in Example 3
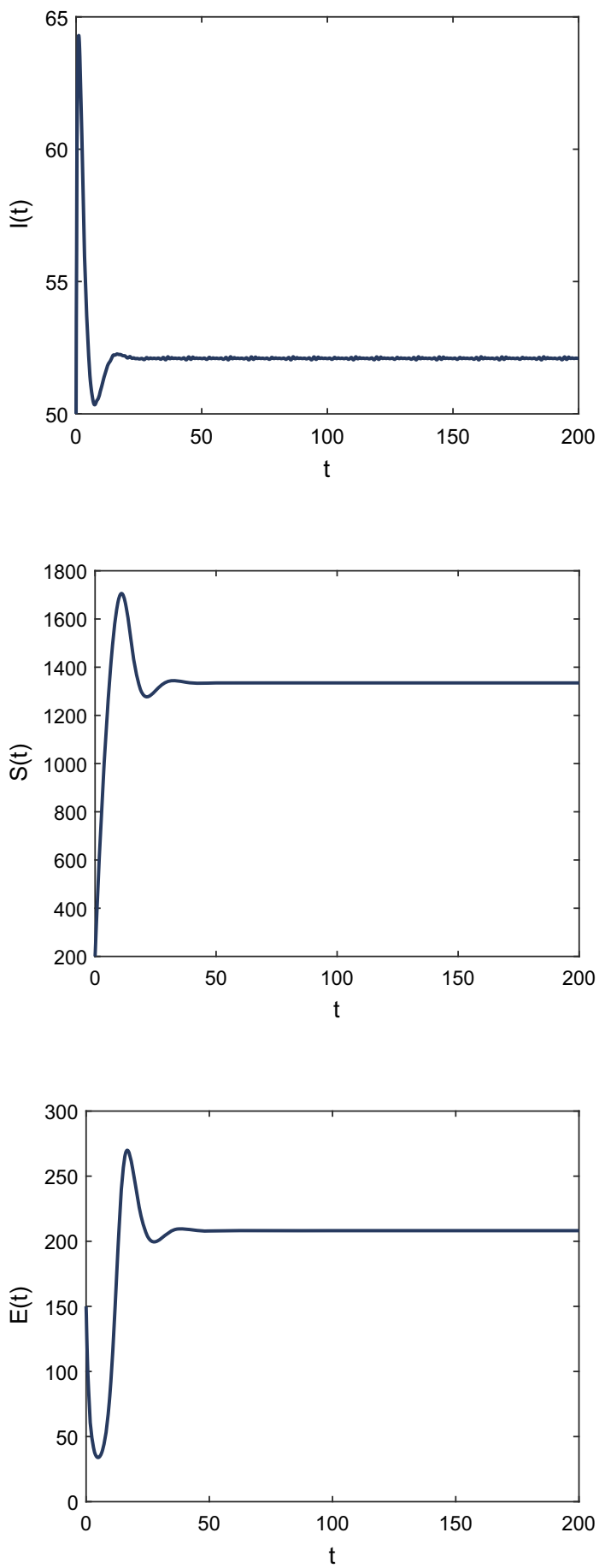
Fig. 9 Time series plot of the infective population for

$\mathcal{R}_{0}=2.6042>1$ with initial conditions, parameter values as in Example 3

Fig. 10 Time series plot of the susceptible population for $\mathcal{R}_{0}=0.7292<1$ with initial conditions, parameter values as in Example 4

Fig. 11 Time series plot of the exposed population for $\mathcal{R}_{0}=0.7292<1$ with initial conditions, parameter values as in Example 4
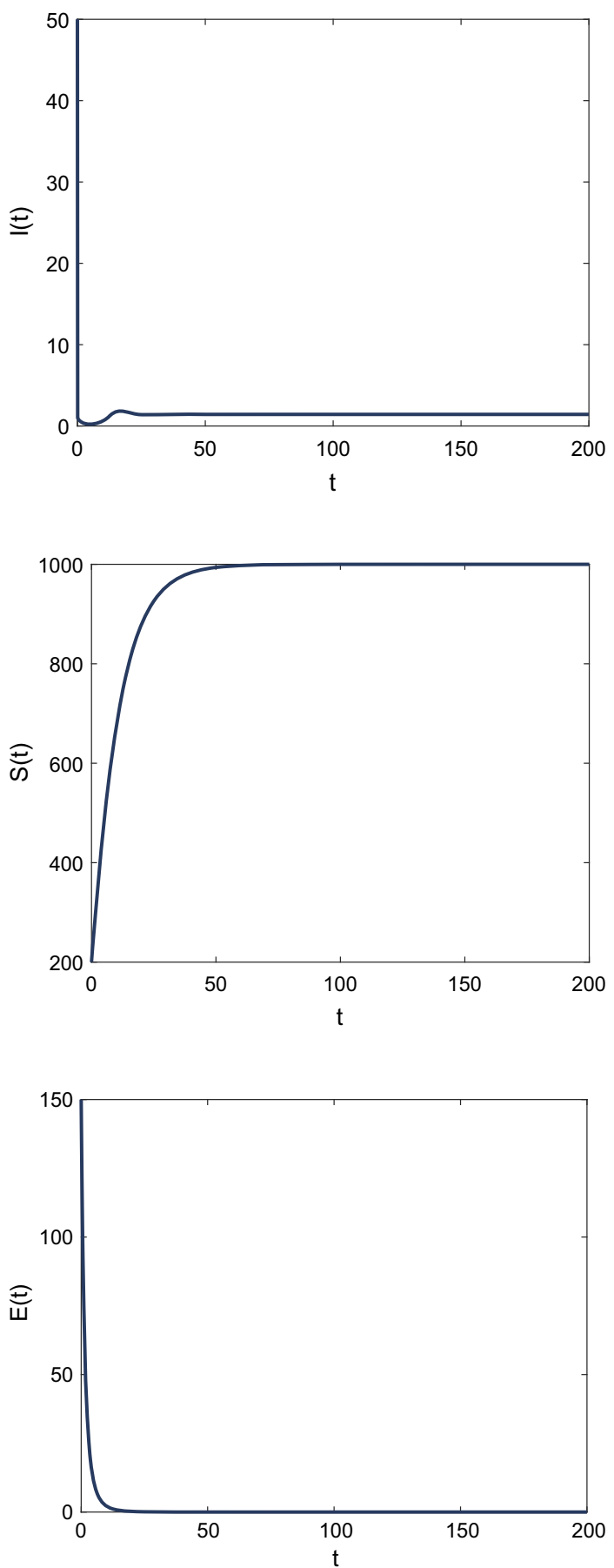
Fig. 12 Time series plot of the infective population for $\mathcal{R}_{0}=0.7292<1$ with initial conditions, parameter values as in Example 4

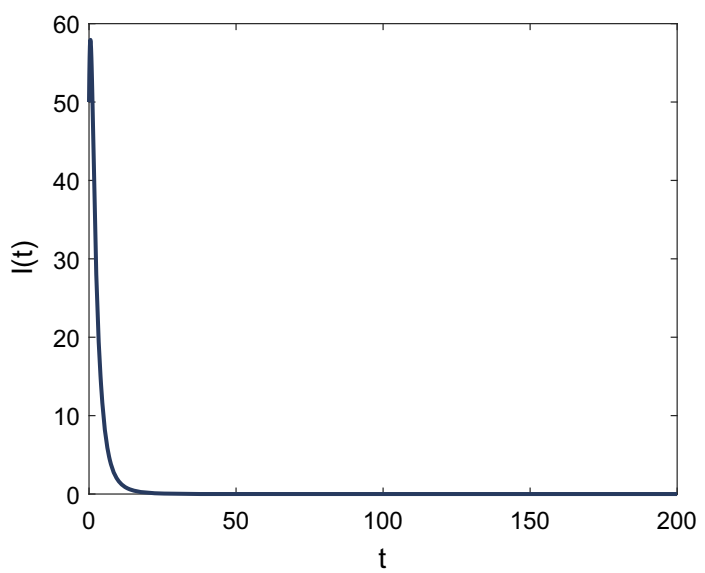

\section{Conclusions}

This paper presented a mathematical study of SEIR epidemiological model with a nonlinear incidence rate and a discontinuous treatment function. Unlike previous SEIR epidemic models where treatment functions are assumed to be continuous, we are interested in finding the impact of the adoption of a discontinuous treatment function $h(I(t))$ and a nonlinear incidence rate $S(t) g(I(t))$.

The basic reproductive number $R_{0}$ is derived under certain conditions on the nonlinear incidence rate and discontinuous treatment function. It is a sharp threshold value to determine the global dynamical behaviors of system (2.4). When $R_{0} \leq 1$, the disease-free equilibrium $P_{0}$ is globally stable so that the disease goes to extinction, while if $R_{0}>1$, the disease-free equilibrium is unstable and the endemic equilibrium $P^{*}$ is globally stable so that the disease remains endemic. Simulation results in above section explained the influence of the choice of the parameters, treatment function or nonlinear incidence rate on Theorem 3.4 or Theorem 3.5. A true advantage of discontinuous treatments is the global convergence to the diseasefree equilibrium $P_{0}$ in finite time. This suggests that from the view point of eliminating the disease, discontinuous treatments would be superior to the continuous ones.

A good model must be simple enough to be mathematically tractable, but complex enough to represent a system realistically. It is worth noting that one of the shortcomings of our model is its reliance on a special kind of incidence rates. The assumptions of Theorems 3.4 and 3.5 are directly linked to this incidence rate. The choice of a more generalized incidence rate will be challenging and we leave this as a further project.

Acknowledgements The authors are grateful to the editors and reviewers whose helpful comments and suggestions have led to much improvement of the paper.

\section{References}

1. Baciotti, A., Ceragioli, F.: Stability and stabilization of discontinuous systems and non-smooth Lyapunov function. ESAIM Control Optim. Calc. Var. 4, 361-376 (1991)

2. Brauer, F.: Epidemic models with heterogeneous mixing and treatment. Bull. Math. Biol. 70, 1869-1885 (2008) 
3. Capasso, V., Serio, G.: A generalization of the Kermack-Mckendrick deterministic epidemic model. Math. Biosci. 42, 41-61 (1978)

4. Filippov, A.F.: Differential Equations with Discontinuous Righthand Sides, Mathematics and Its Applications (Soviet Series). Kluwer Academic, Boston (1988)

5. Gao, D.P., Huang, N.J.: A note on global stability for a tuberculosis model. Appl. Math. Lett. 73, 163-168 (2017)

6. Gao, D.P., Huang, N.J.: Optimal control analysis of a tuberculosis model. Appl. Math. Model. 58, 47-64 (2018)

7. Gao, D.P., Huang, N.J., Kang, S.M., Zhang, C.: Global stability analysis of an SVEIR epidemic model with general incidence rate. Bound. Value Problems 2018, 42 (2018)

8. Guo, Z.Y., Huang, L.H., Zou, X.F.: Impact of discontinuous treatments on disease dynamics in an SIR epidemic model. Math. Biosci. Eng. 9, 97-110 (2012)

9. Hethcote, H.W., Van Den Driessche, P.: Some epidemiological models with nonlinear incidence. J. Math. Biol. 29, 271-287 (1989)

10. Hu, Z.X., Bi, P., Ma, W.B., Ruan, S.G.: Bifurcations of an SIRS epidemic model with nonlinear incidence rate. Discrete Contin. Dyn. Syst. Ser. B 15, 93-112 (2011)

11. Huang, L.H., Guo, Z.Y., Wang, J.F.: Theory and Applications of Differential Equations with Discontinous Righthand Sides. Science Press, Beijing (2011)

12. Kar, T.K., Batabyal, A.: Modelling and analysis of an epidemic model with non-monotonic incidence rate under treatment. J. Math. Res. 2, 103-115 (2010)

13. Korobeinikov, A., Maini, P.K.: A Lyapunov function and global properties for SIR and SEIR epidemiological models with nonlinear incidence. Math. Biosci. 1, 57-60 (2004)

14. Korobeinikov, A., Maini, P.K.: Nonlinear incidence and stability of infectious disease models. Math. Med. Biol. 22, 113-128 (2005)

15. Li, X.Z., Li, W.S., Ghosh, M.: Stability and bifurcation of an SIR epidemic model with nonlinear incidence and treatment. Appl. Math. Comput. 210, 141-150 (2009)

16. Sharomi, O., Gumel, A.B.: Curtailing smoking dynamics: a mathematical modelling approach. Appl. Math. Comput. 195, 475-499 (2008)

17. Shen, M.W., Xiao, Y.N.: Global stability of a multi-group SVEIR epidemiological model with the vaccination age and infection age. Acta. Appl. Math. 144, 137-157 (2016)

18. Wang, L.W., Liu, Z.J., Zhang, X.G.: Global dynamics for an age-structed epidemic model with media impact and incomplete vaccination. Nonlinear Anal. Real World Appl. 32, 136-158 (2016)

19. Wang, W.D.: Backward bifurcation of an epidemic model with treatment. Math. Biosci. 201, 58-71 (2006)

20. Wang, W.D., Ruan, S.G.: Bifurcations in an epidemic model with constant removal rate of the infectives. J. Math. Anal. Appl. 291, 775-793 (2004)

21. Xiao, D.M., Ruan, S.G.: Global analysis of an epidemic model with nonmonotone incidence rate. Math. Biosci. 208, 419-429 (2007)

22. Yang, M., Sun, F.Q.: Global stability of SIR models with nonlinear incidence and discontinuous treatment. Electron. J. Diff. Equ. 304, 1-8 (2015)

23. Zhang, T.L., Kang, R.N., Wang, K., Liu, J.L.: Global dynamics of an SEIR epidemic model with discontinuous treatment. Adv. Diff. Eq. 361, (2015). https://doi.org/10.1186/s13662-015-0695-0

Publisher's Note Springer Nature remains neutral with regard to jurisdictional claims in published maps and institutional affiliations. 